\title{
When two heads are not better than one: Partner neglect in paired memory tasks
}

\author{
Olivia K.A. Lima, Vikram K. Jaswal, and Chad S. Dodson \\ University of Virginia, Charlottesville, Virginia
}

\begin{abstract}
People can monitor the accuracy of their own memories and regulate their responses accordingly. But can they monitor and make use of another person's memory? We document a new phenomenon whereby participants neglect a partner's expertise when deciding whether to defer to that partner's memory or to rely on their own. In two experiments, participants studied images for more time than, less time than, or the same amount of time as a partner, and on subsequent recognition tests they were directed to maximize team performance by either answering themselves or letting their partner respond. In both experiments, individuals failed to use the knowledge that the partner would probably have a better memory for certain items. Only when explicitly instructed to estimate their accuracy relative to their partner's did participants take advantage of the partner's greater expertise.
\end{abstract}

From time to time, we all must consider the accuracy of our own memory, relative to that of another's. As an extreme example, consider the game show Who Wants to Be a Millionaire, in which a contestant has the option of phoning a friend when unsure of the answer to a question. Assuming he or she had an answer in mind before using this "lifeline" option, the contestant must weigh his or her own guess against the friend's and maximize the probability of success by submitting the answer likely to be correct. Success depends, in part, on two components: (1) monitoring the accuracy of one's own memory (i.e., recognizing when one is sufficiently uncertain to call a friend) and (2) assessing the relative accuracy of another's response (i.e., recognizing another's greater expertise). While there is much research on people's abilities to monitor the accuracy of their own memories (e.g., Kelley \& Sahakyan, 2003; Koriat \& Goldsmith, 1996), to our knowledge no one has investigated the second component, that is, assessing the accuracy of others' memories. Such an ability would, however, be advantageous. Apart from improving their chances of succeeding in game shows, people who excel at monitoring others' memories stand to be efficient decision makers who know when to seek out and make use of others' greater knowledge.

With respect to the first component, two lines of evidence indicate that people are generally good at monitoring the accuracy of their own memories. First, confidence ratings in the accuracy of a response tend to correlate well with actual accuracy, except for items designed to create false memories (e.g., Kelley \& Sahakyan, 2003). Second, Koriat and Goldsmith (1996) have shown that people can control and regulate overall memory accuracy by withholding answers of questionable accuracy. For example, on both recognition and recall tests involving general knowledge questions, participants substantially improved overall accuracy when given the option to withhold lowconfidence answers, compared to when they were forced to answer all of the test questions.

The present article investigates one's ability to regulate performance when the second component is required: monitoring the accuracy of a partner's memory relative to one's own. Participants encoded pictures that were displayed for either a brief $(400-\mathrm{msec})$ or long $(5,000$ msec) duration, and were told their partner had studied the images for either the same or the opposite amount of time. On subsequent recognition tests, participants were directed to maximize performance by submitting either their own response or their partner's. We are interested in participants' ability to regulate performance when they have studied images for opposite amounts of time from their partner. Successful regulation in this case depends on inferring that when one's own memory for an item is bad, the partner's memory for that particular item is likely to be good (i.e., a "hard for me = easy for you" strategy). In other words, can participants recognize and take advantage of a partner's relative expertise on particular items?

There is good reason to expect that participants will make this inference, since individuals have been shown to make similar multistep inferences about their own memories. For instance, people can readily infer that the absence of memory for expected information is evidence of an event's nonoccurrence (Dodson \& Schacter, 2002). In addition, recognizing the relative accuracy or fallibility of a partner's memory should simply be a matter of adjusting from one's own memory for the item (Nickerson, 1999). To anticipate our results, however, we find that individuals, to some extent, are unable to make strategic use of a partner's greater expertise.

C.S.Dodson, cdodson@virginia.edu 


\section{EXPERIMENT 1}

Participants and partners watched slideshows of identical images that were presented for differing periods of time. Over three study blocks, participants were informed that their partner would study the images for the same amount of time, for the opposite amount of time, or participants were asked to complete the task individually. During a recognition test, participants had the option of submitting their own response or passing to the partner, or in the condition with no partner, of either submitting or withholding a response. The most effective passing strategy would require participants to compare the likely accuracy of their own memory with that of the partner's.

\section{Method}

Participants. Sixteen undergraduates, 10 women and 6 men (mean age: 18.5), participated for course credit.

Stimuli. The stimuli consisted of 166 color images of familiar objects, such as shoes and elephants, taken from a digital library of photo objects (Hemera Technologies) and sized to fit within a $4 \times 4$ in. square. No two images from the same basic-level category were used (see Rosch, Mervis, Gray, Johnson, \& Boyes-Braem, 1976, for more details about basic-level categories). Recognition testing was conducted with a three-ring binder of printed color arrays, each containing one target stimulus and three similar distractors from the same basic-level category, arranged in the four quadrants of an $8.5 \times 11$ in. piece of paper (see Figure 1).

Procedure. Each participant was introduced to a confederate whom they were led to believe was a fellow participant. They sat on opposite sides of a table, with a PowerBook G3 laptop in front of the participant and an iBook G3 laptop in front of the confederate. The experimenter informed the pair they would work together in a cooperative memory game, watching slideshows of images on their respective laptops and attempting to identify each image from an array of similar ones. As an example, a sample image and array (as in Figure 1) were shown. The team would work together, earning 1 point for every correct answer submitted as a team and losing 1 point for every incorrect answer submitted. At the end of the study,

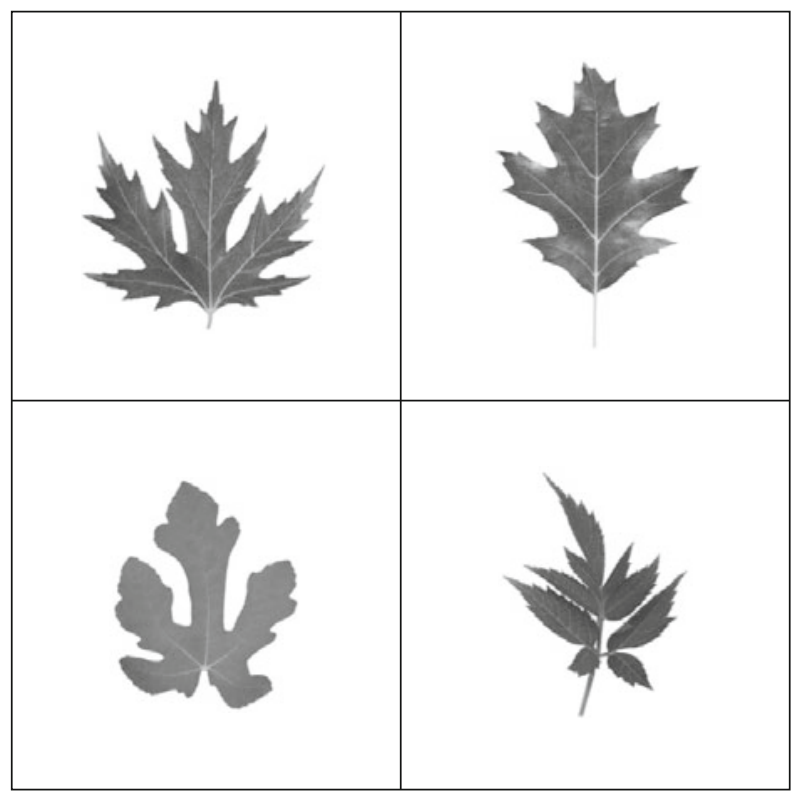

Figure 1. Sample test array. the amount of candy they would receive depended on their score. Participants were informed that the task would require their close attention to detail.

The memory game consisted of three separate blocks of trials: Same, Opposite, and Individual. Each block had an encoding phase, a distractor task, and a recognition test. The encoding phase consisted of 24 target images displayed sequentially, using PsyScope (Cohen, MacWhinney, Flatt, \& Provost, 1993): half for a short period of time $(400 \mathrm{msec})$ and half for a long period $(5,000 \mathrm{msec})$. In addition, 26 images, half short, half long, were included at the beginning (13) and end (13) of each encoding phase, as buffers to reduce primacy and recency effects. The order of the images in each block was determined randomly at first, and held constant across participants. The order of short-duration and long-duration images was also randomly determined, with the constraint that a maximum of four short or four long images appear in a row. Two versions of each list were created to counterbalance the duration of specific items across subjects.

The encoding phase varied across the three blocks only by the timing of the partner's slideshows. In the Same block, participants were informed that their partner would see the same images for the same amount of time as they did; items seen for a short time by the participants would also be seen for a short time by the partner, and likewise for the long items. In the Opposite block, participants and their partner would again see the same images, but now for opposite amounts of time, so that images seen for a short time by the participant would be seen for a long time by the partner, and vice versa. Finally, in the Individual block, only the participant watched the slideshow, while the partner's laptop remained closed. The Individual block always occurred between the Same and Opposite blocks, while half the participants had the Same block first and half the Opposite. Participants were informed prior to each encoding phase whether their partner would see the images for the same or opposite amount of time, and were also reminded of this prior to testing. In a manipulation check after the paired blocks, all participants were able to correctly report their partner's exposure times.

After a distractor task, in which participants and partners spent 5 min circling differences in pairs of drawings, the recognition testing began. Participants were shown 24 arrays of images from the immediately preceding encoding phase, 12 of which had been seen by the participant for a short time and 12 for a long time. One target was presented per array, along with three distractors (see Figure 1). The location of the targets in the array was randomly determined, with the constraint that, in a block, they had to appear equally often in each quadrant. The order of the arrays within the block was also randomly determined, and held constant across participants.

Participants were shown one array at a time and were asked to point to the image they had seen in the slideshow. Although participants always had to indicate a choice, they also had the option of withholding this answer, and in the Same and Opposite blocks would then pass the array to the partner, whose answer would be submitted for the team. They were informed that the number of passes did not matter, but rather that they should maximize the total number of correct answers submitted. As a team, they received 1 point for every correct answer submitted and lost 1 for every incorrect answer. Importantly, the pair could not discuss the items, and an occluder prevented them from seeing each other's choices. Since no feedback was available, participants could therefore decide only to submit or pass, on the basis of their knowledge about the timing of the slideshow their partner had seen. In the Individual block, participants had the option of submitting or withholding their answers, with the same scoring system for submitted answers and no penalty for withheld answers.

To ensure that participants understood the procedure, a short practice block was administered at the beginning of the study, similar to the Individual block but with only 16 images at encoding and 8 at testing ( 4 short and 4 long), as well as a 2 -min distractor task. No feedback was given after this block, but participants were asked if they had questions before proceeding with the experimental blocks. 
At the end of the study, participants were asked directly whether they thought it was easier to remember images seen for a short or long time.

\section{Results}

The primary result was that participants were surprisingly indifferent to their partner's experiences. Even though during the Opposite block the partner had seen some items for 12 times as long as the participant, participants were unable to capitalize on that additional experience.

Overall accuracy. We first confirmed that participants were significantly more accurate in recognizing items seen for 5,000 msec than those seen for $400 \mathrm{msec}$. We calculated their overall accuracy without considering whether they chose to pass. As Table 1 shows, across blocks participants correctly recognized $78 \%$ of long items but only $55 \%$ of short items. A 3 (block) $\times 2$ (duration) ANOVA on these data confirmed a significant effect of duration $[F(1,15)=81.69, p<.0001]$, with no effect of block and no interaction $(F \mathrm{~s}<1)$.

Passing. Next, we analyzed passing rates. As Table 1 shows, participants passed more short than long items, which is not surprising given the accuracy results, and they did so consistently across all three blocks. A 3 (block) $\times$ 2 (duration) ANOVA on the number of passes confirmed a significant effect of duration $[F(1,15)=53.47, p<$ $.0001]$. There was also a main effect of block $[F(2,30)=$ $4.41, p<.05]$, such that participants passed fewer items in the Individual block than they passed in the Same and Opposite blocks (Tukey's HSD, $p<.05$ ), which did not differ from each other. This greater tendency to pass to a partner is likely due to factors of social demand unrelated to the memory questions at hand.

One might expect participants to pass more short items and fewer long items in the Opposite block than in the Same block. After all, for Opposite short items, not only was participants' accuracy low, but they could reasonably expect their partner's accuracy to be high (vice versa for the long items). However, there was no significant interaction between block and duration $(F<1)$. In the Same block, participants passed $51 \%$ of the short items and $22 \%$ of the long; in the Opposite block, they passed 55\% of the short items and $19 \%$ of the long.

Improvement in accuracy. Although participants passed the same numbers of items in the Same and Oppo-

Table 1

Overall Accuracy and Items Passed by Block and Item Duration in Experiment 1

\begin{tabular}{|c|c|c|c|c|c|c|}
\hline & \multicolumn{6}{|c|}{ Block } \\
\hline & \multicolumn{2}{|c|}{ Individual } & \multicolumn{2}{|c|}{ Same } & \multicolumn{2}{|c|}{ Opposite } \\
\hline & $M$ & $S E$ & $M$ & $S E$ & $M$ & $S E$ \\
\hline \multicolumn{7}{|c|}{ Overall Accuracy (\%) } \\
\hline Short & 55.6 & 5.0 & 56.9 & 4.2 & 51.6 & 4.0 \\
\hline Long & 77.6 & 4.4 & 77.7 & 4.4 & 76.7 & 3.8 \\
\hline \multicolumn{7}{|c|}{ Items Passed (\%) } \\
\hline Short & 41.7 & 6.0 & 50.8 & 5.5 & 55.0 & 4.4 \\
\hline Long & 15.8 & 3.6 & 21.7 & 4.7 & 19.2 & 3.6 \\
\hline
\end{tabular}

Note-Short items were studied for $400 \mathrm{msec}$; long, for 5,000 msec. site blocks, they may have passed more strategically in the latter. For example, participants who passed often could improve their accuracy if they passed a greater number of incorrect than correct items. Therefore, we also calculated a measure of improvement in accuracy. Here participants' overall accuracy, based on responses to all items, was subtracted from their accuracy on submitted items alone, yielding the number of percentage points they improved through passing. ${ }^{1}$ Simply being given the opportunity to pass should result in an improvement in accuracy across all blocks (Koriat \& Goldsmith, 1996). However, if participants were making strategic use of their partner's experiences, we would expect the greatest improvement in accuracy in the Opposite block, particularly for items the participants had seen for a short duration because their partner had seen them for a long duration.

As Figure 2 shows, the opportunity to pass or withhold resulted in improvement for all blocks, as expected, but the predicted additional improvement on short trials in the Opposite block did not occur. A 3 (block) $\times 2$ (duration) ANOVA on the improvement in accuracy scores revealed no main effects and no interaction $(F \mathrm{~s}<1.5)$. Participants did not take advantage of their partner's experiences in the Opposite block in order to improve their accuracy beyond what could be gained merely from withholding responses in either the Same or the Individual blocks.

Loss in quantity. The improvement in accuracy score does not distinguish between participants who improve by passing incorrect responses only (preserving all of their correct choices), and those who discard both correct and incorrect responses. In order to measure this possible loss of correct responses, following Koriat and Goldsmith (1996) we computed a "quantity-loss" score for each individual. The number of correct responses submitted was subtracted from the overall number of correct responses (provided before the option to pass or withhold); this is reported as a percentage of total items.

Participants' quantity-loss scores for short items were $19 \%, 26 \%$, and $24 \%$ for the Individual, Opposite, and Same blocks, respectively. Their quantity-loss scores for the long items in the three blocks were $5 \%, 11 \%$, and $11 \%$, respectively. A 3 (block) $\times 2$ (duration) ANOVA on these quantity-loss scores showed only a main effect of duration $[F(1,15)=21.6, p<.01]$, indicating that a consequence of passing was a greater loss in the quantity of correct responses for the short items (which were most often passed) than for the long items. However, as with the improvement in accuracy scores, there were no differences between the Opposite and Same blocks in the number of correct responses lost from passing.

In sum, we had expected that participants would improve more in the Opposite block than in the Same block, specifically on short items, for which partners had greater experience to offer. This did not occur; there were no differences between the blocks in terms of either the improvement-in-accuracy score or the quantity-loss score. Importantly, this lack of difference in performance between the Opposite and Same blocks cannot be attributed to participants' misunderstanding their partner's experiences, since all participants were able to correctly report 

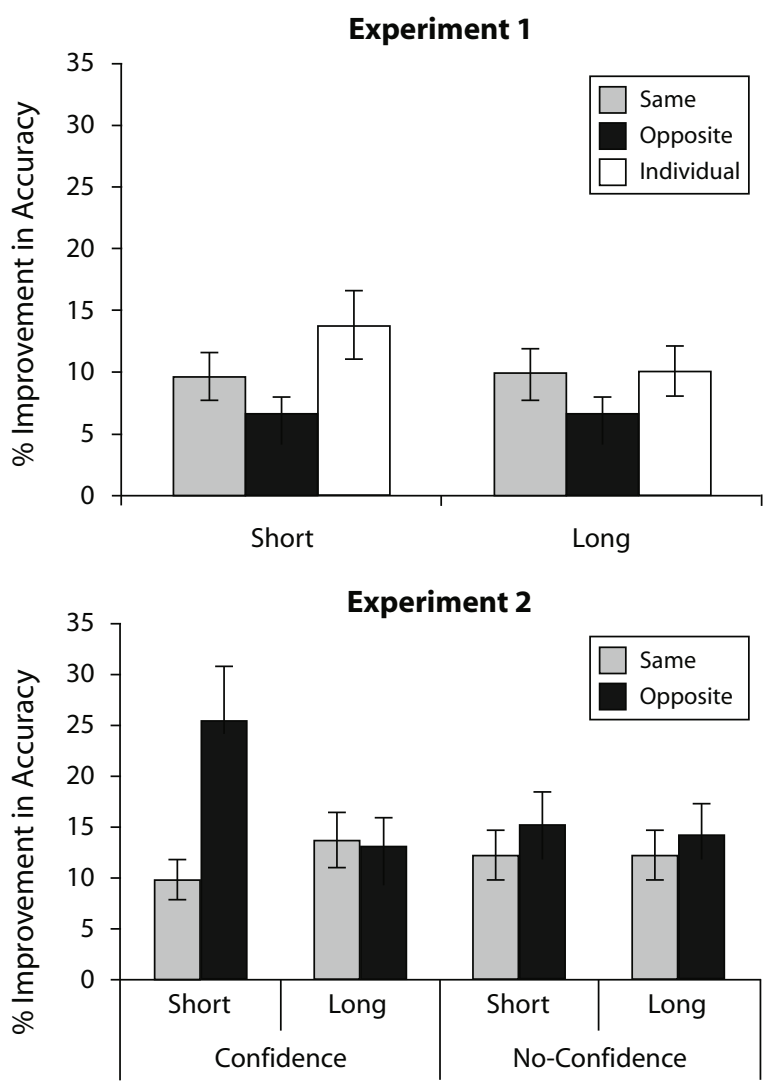

Figure 2. Improvement in accuracy as a function of block, duration, and condition in Experiments 1 and 2.

this after each block. Additionally, when asked directly, participants were aware of the effect experience had on accuracy, with all but 2 participants reporting that long items were easier to recall than short items.

One concern is that during the test participants may not have remembered on an item-by-item basis whether they or their partner had seen the item for $400 \mathrm{msec}$ or 5,000 msec during the encoding stage. However, participants passed more than twice as many short as long items, and so it seems reasonable to conclude that they were less certain about short than long items and used this to guide their behavior (see Koriat \& Goldsmith, 1996). But they were unable to integrate spontaneously what their own lack of certainty implied about the likely accuracy of their partner's memory. This provided the motivation for Experiment 2 , in which we required participants to explicitly consider their confidence in relation to their partner's.

\section{EXPERIMENT 2}

In Experiment 1, participants were surprisingly inattentive to their partner's experience-experience that might have led to better performance on certain items. Improvement in accuracy on items for which the partner had 12 times more experience was identical to improvement when the partner had the same (brief) exposure. One explanation of these findings is that participants based the decision to pass exclusively on their own sense of ac- curacy, without inferring what this might mean about the accuracy of their partner's memory.

To encourage participants to consider their partner's experience, we introduced a new condition. Prior to each decision to pass or submit, participants in a Confidence condition rated how confident they were in the accuracy of their own response, and also predicted how confident their partner would be in the accuracy of her response, if the item were passed. Our rationale was that participants might pass more strategically if directed to compare the likely accuracy of their own and their partner's memory for an item. This would result in a greater improvement in accuracy when passing short items in the Opposite block (where the partner had greater expertise) than in the Same block (where the partner had no particular expertise to offer). A second group of participants (No-confidence) did not make confidence judgments, and so was expected to show the same pattern as found in Experiment 1.

\section{Method}

Participants. Seventeen men and 23 women (mean age: 21.5 years), all members of the university community, participated for course credit or $\$ 10$. Participants were randomly assigned to Noconfidence or Confidence conditions.

Procedure. The experiment used the same stimuli as did Experiment 1 . The Individual block was removed because we were only interested in the comparison between the partnered blocks. Participants thus completed only two experimental blocks, Same and Opposite. For these blocks, participants in the No-confidence condition followed exactly the same procedure as in Experiment 1.

Participants in the Confidence condition followed a similar procedure, but they also gave confidence ratings during each recognition trial. After pointing to the item they believed had been presented in the encoding phase, participants indicated how sure they were of their accuracy on a scale from 1 to 4 , where 1 was a complete guess and 4 absolute certainty (this choice was made on a paper scale hidden by an occluder). Participants also used the same scale to estimate the likely accuracy of their partner's response if the item were passed. Only after both confidence ratings were made did participants inform the experimenter of their decisions to pass or submit the answers.

\section{Results}

The main result was that participants in the Confidence condition succeeded in making use of their partner's experience during the Opposite block to improve performance. Those in the No-confidence condition, like those in Experiment 1 , did not.

Overall accuracy. As Table 2 shows, participants' overall accuracy for all blocks was again greater for items seen for 5,000 msec than for $400 \mathrm{msec}$, replicating Experiment 1 . A 2 (block) $\times 2$ (duration) $\times 2$ (condition) ANOVA of overall accuracy yielded a significant main effect of duration $[F(1,38)=153.49, p<.0001]$, with no other main effects or interactions $\left(F_{\mathbf{S}}<3\right)$.

Passing. Table 2 shows that, as in Experiment 1, participants passed more short than long items $[F(1,38)=$ $82.90, p<.0001]$. However, by contrast to Experiment 1 , where participants passed the same number of items in the Opposite and Same blocks, in Experiment 2 they passed more items in the Opposite block $[F(1,38)=30.78, p<$ $.0001]$. Also by contrast to Experiment 1, an interaction between block and duration showed that short items in 
Table 2

Overall Accuracy and Items Passed by Condition, Block, and Item Duration in Experiment 2

\begin{tabular}{|c|c|c|c|c|c|c|c|c|}
\hline & \multicolumn{4}{|c|}{ No-Confidence } & \multicolumn{4}{|c|}{ Confidence } \\
\hline & \multicolumn{2}{|c|}{ Same } & \multicolumn{2}{|c|}{ Opposite } & \multicolumn{2}{|c|}{ Same } & \multicolumn{2}{|c|}{ Opposite } \\
\hline & $M$ & $S E$ & $M$ & $S E$ & $M$ & $S E$ & $M$ & $S E$ \\
\hline \multicolumn{9}{|c|}{ Overall Accuracy (\%) } \\
\hline Short & 52.1 & 2.8 & 48.3 & 4.2 & 56.2 & 3.1 & 48.3 & 3.8 \\
\hline Long & 79.2 & 3.1 & 75.8 & 3.6 & 72.9 & 4.4 & 75.4 & 3.8 \\
\hline \multicolumn{9}{|c|}{ Items Passed (\%) } \\
\hline Short & 45.8 & 3.2 & 60.8 & 3.3 & 65.8 & 3.6 & 77.5 & 2.3 \\
\hline Long & 25.8 & 3.2 & 27.5 & 4.4 & 32.5 & 4.3 & 32.5 & 5.1 \\
\hline
\end{tabular}

Note-Short items were studied for $400 \mathrm{msec}$; long, for 5,000 msec.

particular were passed more often in the Opposite block $[F(1,38)=9.41, p<.01]$. Although we had not expected these differences from Experiment 1, we speculate that they were caused by the removal of the intervening Individual block, which heightened the contrast between the paired blocks. Finally, participants passed more items in the Confidence than No-confidence conditions $[F(1,38)=$ $12.81, p<.001]$. There were no other significant effects or interactions.

Improvement in accuracy. Improvement-in-accuracy scores are shown in Figure 2. ${ }^{2}$ Replicating Experiment 1, participants in the No-confidence condition improved similar amounts on short items in the Same and Opposite blocks (13\% vs. 16\%). Participants in the Confidence condition, by contrast, improved more than twice as much on short items in the Opposite block over short items in the Same block (28\% vs. $12 \%)$. In contrast to improvement on short items, participants' improvement in accuracy on long items did not differ between the Confidence and No-confidence conditions or across the Same and Opposite blocks.

A three-way ANOVA on the improvement data revealed only this three-way interaction $[F(1,28)=5.63, p<.05]$. Importantly, participants' greater improvement in the Confidence condition on short items in the Opposite but not the Same block indicates that it was not the mere fact that they were making confidence ratings that enabled them to pass more strategically. Making confidence ratings was beneficial only in the Opposite block because that was the one where participants could use the "hard for me = easy for you" heuristic.

Loss in quantity. Given the higher rates of passing in the Confidence condition, it is possible that this group's marked improvement in accuracy on Opposite versus Same short items came at the expense of a greater loss in quantity, as compared to the No-confidence condition. This, however, was not the case. In the Confidence condition the quantity-loss score for short items was $27 \%$ in the Opposite block and 30\% in the Same block, which do not differ from each other $[t(13)=1.20$, n.s. $]$. Likewise, in the No-confidence condition, there were comparable quantity-loss scores for short items in the Opposite (25\%) and Same $(17 \%)$ blocks $[t(15)=1.80$, n.s. $]$.

Confidence ratings. We have argued that participants in the Confidence condition showed greater improve- ment in accuracy on short items in the Opposite block because they correctly evaluated their partner's likely accuracy relative to their own. This assumption is easily verified by examining the confidence ratings, shown in Table 3, with difference scores (partner confidence minus self-confidence) in the last row. A 2 (block) $\times 2$ (duration) ANOVA on the difference scores yielded a significant effect of duration $[F(1,19)=36.10, p<.0001]$ and a significant interaction $[F(1,19)=19.04, p<.001]$. In the Same block, there was a general tendency for participants to overestimate their partner's likely accuracy relative to their own for items of both durations. By contrast, in the Opposite block, participants appropriately rated their partner's accuracy as higher than their own for items the participants saw for $400 \mathrm{msec}$ (and their partner saw for 5,000 $\mathrm{msec}$ ), and lower for items the participants saw for 5,000 msec (and their partners saw for $400 \mathrm{msec}$ ). This pattern in the Opposite block indicates that participants not only correctly evaluated their partner's likely accuracy relative to their own but attributed it to length of exposure (a factor that varied systematically for the partners), as opposed to any other item-intrinsic quality, such as difficulty.

In sum, orienting participants to evaluate the accuracy of their own memories relative to their partner's led to an average improvement-in-accuracy score on Opposite short items more than twice as great as the average improvement-in-accuracy score on Same short items. By contrast, participants in the No-confidence condition, as in Experiment 1, showed the same (relatively small) amount of improvement in accuracy across the Opposite and Same blocks. Despite these large differences in the improvement-in-accuracy scores, both the Confidence and No-confidence conditions showed comparable increases in the passing rate from the Same to the Opposite blocks, accompanied by nonsignificant changes in the quantity of correct items that were lost. Therefore, directing participants to give confidence ratings in a context where the partner is expected to have greater expertise on certain items appears to improve participants' ability to discriminate correct from incorrect responses.

\section{DISCUSSION}

In two experiments, participants studied images for either the same or the opposite amounts of time as did a partner, and on subsequent recognition tests they were directed to maximize performance by submitting either their own or their partners' responses. The key finding from both experiments is that individuals fail to achieve tangible gains from their partner's greater experience unless explicitly required to consider it. That is, participants show comparable increases in the accuracy of their responses when passing to a partner who has studied an item for the same amount of time as themselves, as when passing to one who has studied an item for over 12 times longer. Only when participants are asked explicitly to compare the accuracy of their own and their partner's memory do they improve in strategic use of the latter.

This neglect of a partner's expertise is remarkable for a number of reasons. First, how long an item was stud- 
Table 3

Confidence Ratings for Self and Partner by Block in Experiment 2

\begin{tabular}{|c|c|c|c|c|}
\hline & \multicolumn{4}{|c|}{ Block } \\
\hline & \multicolumn{2}{|c|}{ Same } & \multicolumn{2}{|c|}{ Opposite } \\
\hline & $M$ & $S E$ & $M$ & $S E$ \\
\hline \multicolumn{5}{|c|}{ Short } \\
\hline Self & 2.2 & 0.10 & 2.1 & 0.10 \\
\hline Partner & 2.7 & 0.13 & 3.0 & 0.10 \\
\hline Difference & -0.4 & 0.09 & -0.9 & 0.14 \\
\hline \multicolumn{5}{|c|}{ Long } \\
\hline Self & 3.0 & 0.10 & 3.0 & 0.13 \\
\hline Partner & 3.1 & 0.11 & 2.6 & 0.12 \\
\hline Difference & -0.1 & 0.09 & 0.4 & 0.17 \\
\hline
\end{tabular}

Note-Confidence scale ranged from 1 (guessing) to 4 (certainty). Short items were studied for $400 \mathrm{msec}$; long, for 5,000 $\mathrm{msec}$.

ied clearly predicted accuracy, for participants were $20 \%$ more accurate at recognizing items seen for a longer duration. Second, participants themselves acknowledged that duration played a role in accuracy; at the end of the study, all but 2 participants asserted that long items were easier to remember. Third, participants were aware of their partner's experience, since all participants were able to report it. Fourth, as the confidence ratings in Experiment 2 showed, participants were capable of inferring partner accuracy when explicitly required to do so (indeed, they even overestimated the relative expertise of their partner). Finally, participants were able to monitor and act upon the likely fallibility of their own memory, as they generally passed more short than long items. Thus, all of the knowledge and skills necessary to capitalize on their partner's experience were in place, yet participants failed to do so successfully unless explicitly required to consider their partner.

We suggest that the cause of this "partner neglect" phenomenon involves the general problem of perspectivetaking. Put simply, participants are unable to benefit from their partner's expertise because it is not salient to them. The likely accuracy of one's own memory is much more apparent than is the estimated accuracy of another's, even in situations where one could use a "hard for me = easy for you" strategy. In some respects, our findings are foreshadowed by Flavell (1977), who noted that, "We experience our own point of view more or less directly, whereas we must always attain the other person's view in a more indirect manner. Furthermore, we are usually unable to turn our own viewpoint off completely when trying to infer the other's" (p. 124). Apparently, it is only when we are required to estimate another's knowledge that we make full use of it.

These results are closely related to Koriat and Goldsmith's (1996) finding that individuals can regulate overall memory accuracy by withholding answers of which they are unsure. A number of variables have been shown to affect this regulatory ability, including task characteristics, such as the penalty for making a mistake, and participant characteristics, with older adults showing poorer regulation (e.g., Kelley \& Sahakyan, 2003). Interestingly, our results indicate that the ability to monitor accuracy and regulate responses accordingly does not spontaneously extend to considering another person in group memory situations.

Calling upon another person's memory greatly increases the amount of information we have at our disposal (Wegner, 1995). However, making effective use of this transactive memory network requires exactly the kind of monitoring of one's own and another's memories that we have shown may not occur spontaneously. Interestingly, participants in collaborative memory tasks who discuss and jointly attempt to remember material do spontaneously divide material according to stable person characteristics, such as expertise in a given domain (Hollingshead, 1998; Wegner, 1995). Our task, however, was not collaborative, as participants alone had to decide whether to submit their responses or their partner's. Further, they had to do so based only on a situational characteristic - namely, whether they had seen an item for a longer or shorter time than had their partner. Our results indicate that people do not spontaneously consider their partner's situational experience relative to their own, and therefore do not benefit even from such relevant information as a partner's twelvefold advantage in study time.

\section{AUTHOR NOTE}

Address correspondence concerning this article to C. S. Dodson, Department of Psychology, University of Virginia, Charlottesville, VA 22904 (e-mail: cdodson@virginia.edu).

\section{REFERENCES}

Cohen, J. D., MacWhinney, B., Flatt, M., \& Provost, J. (1993). PsyScope: An interactive graphic system for designing and controlling experiments in the psychology laboratory using Macintosh computers. Behavior Research Methods, Instruments, \& Computers, 25, 257-271.

Dodson, C. S., \& Schacter, D. L. (2002). Aging and strategic retrieval processes: Reducing false memories with a distinctiveness heuristic. Psychology \& Aging, 17, 405-415.

Flavell, J. H. (1977). Cognitive development. Englewood Cliffs, NJ: Prentice Hall.

Hollingshead, A. B. (1998). Communication, learning, and retrieval in transactive memory systems. Journal of Experimental Social Psychology, 34, 423-442.

Kelley, C. M., \& Sahakyan, L. (2003). Memory, monitoring, and control in the attainment of memory accuracy. Journal of Memory \& Language, 48, 704-721.

Koriat, A., \& Goldsmith, M. (1996). Monitoring and control processes in the strategic regulation of memory accuracy. Psychological Review, 103, 490-517.

Nickerson, R. S. (1999). How we know-and sometimes misjudgewhat others know: Imputing one's own knowledge to others. Psychological Bulletin, 125, 737-759.

Rosch, E. S., Mervis, C. B., Gray, W. D., Johnson, D. M., \& BoyesBraem, P. (1976). Basic objects in natural categories. Cognitive Psychology, 8, 382-439.

WEGNER, D. M. (1995). A computer network model of human transactive memory. Social Cognition, 13, 319-339. 


\section{NOTES}

1. For this analysis, the data from 3 participants were excluded: 1 participant passed all 12 short items in the Opposite block and thus had no submitted accuracy to measure; and 2 others achieved $100 \%$ overall accuracy on long items in the Same and/or Opposite block and hence had no room for improvement.

2. For this analysis, the data from 10 participants were excluded: 7 because they achieved $100 \%$ overall accuracy on long items in the Same and/or Opposite block, 2 because they passed all 12 short items in the
Opposite block, and 1 who fit both qualifications. Given this pattern showing a clear differentiation between long and short items and a high level of passes of short items in the Opposite block, had we been able to include these participants, it would only have reinforced the following analyses. The remaining number of participants in the Confidence condition was 14 and in the No-confidence condition was 16 .

(Manuscript received December 4, 2005; revision accepted for publication June 1, 2006.) 